\title{
Special issue on ubiquitous multimedia services
}

\author{
Shiguo Lian • Jean-Henry Morin • Stefanos Gritzalis • \\ Gregory L. Heileman
}

Published online: 28 July 2010

(C) Springer Science+Business Media, LLC 2010

\section{Introduction}

Emerging ubiquitous multimedia services are expected to be available anytime, anywhere, and using different computing devices. Services such as audio/video streaming, digital libraries, on-line businesses, and live camera remote surveillance are widely deployed. Users will be able to access these services anytime, anywhere, and using any computing device in a pervasive way. For example, a user may bring a PDA on a field trip, carry a laptop (with both wireless and wired network cards) on a business trip, use high performance workstations at work, and use desktop PCs at home (with dial-up, cable, or DSL connection). In another example, a user continues to watch the soccer game over home TV, while he watches the game through a mobile device out of home. Some other ubiquitous multimedia services include mobile games and entertainment, mobile blog, mobile community, home network, and so on.

\author{
S. Lian $(\triangle)$ \\ France Telecom R\&D (Orange Labs), \\ Beijing, China \\ e-mail: shiguo.lian@ieee.org \\ J.-H. Morin \\ University of Geneva, \\ Geneva, Switzerland \\ e-mail: Jean-Henry.Morin@unige.ch \\ S. Gritzalis \\ University of the Aegean, \\ Mytilene, Greece \\ e-mail: sgritz@aegean.gr \\ G. L. Heileman \\ University of New Mexico, \\ Albuquerque, USA \\ e-mail: heileman@ece.unm.edu
}

Since ubiquitous multimedia services are constructed on the convergence of various networks (wired or wireless networks, broadcasting or multicasting networks, broadband or band-limited networks, etc.) and devices (PC, PDA, Cell Phone, TV, Camera, etc.), the new challenge in multimedia service management is to deal with heterogeneous client capabilities and dynamic end-to-end resources availability, and to ensure satisfactory service quality for each client. Additionally, privacy, security and ethical issues are becoming increasingly important topics in ubiquitous services.

This special issue of the Information Systems Frontiers aims to expose the readership to the latest research results on ubiquitous multimedia services. It is composed of seven selected papers, covering the interesting topics, e.g., mobile chatting system, ubiquitous surveillance system, personalized news consuming system, home networking systems, etc., demonstrating pioneer work, investigating the novel solutions and discussing the future trends in this field.

\section{The papers in this special issue}

The first paper, "MILC: A Secure and Privacy-Preserving Mobile Instant Locator with Chatting" by (A. Loukas et al. 2010), presents a mobile application scheme, named MILC, that not only supports location-based services and mobile instant messaging but also provides real security and privacy solutions. Thus, the users can chat, interchange geographic coordinates and make Splashes in real-time in control of their own personal information and private sphere. The scheme's architecture, protocols, security and privacy issues are presented and discussed in detail. Additionally, the prototype implemented in Google's Android OS shows the system's good properties. 
In the second paper, "Effective Distributed Service Architecture for Ubiquitous Video Surveillance" by (T.-C. Wang et al. 2010), a distributed and secure architecture for ubiquitous video surveillance (UVS) services is proposed, which is suitable for the condition of Internet or wireless networks. Based on cloud computing, the users can consume UVS services without local physical infrastructure, platform or software. In this scheme, the AES security mechanism, multicast overlay network and forward error correction (FEC) are adopted to protect the service privacy, preserve the service scalability and provide reliable UVS video streaming, respectively. The experimental test-bed is implemented to show the proposed scheme's good performances.

The third paper, "Automatic and Fast Temporal Segmentation for Personalized News Consuming" by (Y. Dong and S. Lian 2010), presents a ubiquitous news program consuming system, which provides the on-demand services to mobile consumers or Internet/home TV consumers. In this system, the automatic news program segmentation and classification is proposed to reorganize the news program according to the news' topics. The core technique, i.e., video temporal segmentation, is introduced, evaluated and compared with existing ones. Experimental results show the proposed system's good performances, such as low computational cost and high correct detection rate.

In the fourth paper, "Advanced Secure Multimedia Services for Digital Homes" by (R. Roman et al. 2010), the secure and ubiquitous digital home system, named Feel@Home, is proposed. This system enables the users to demand access and possibly share such multimedia contents as photographs, videos and music from any location and with any device. Additionally, the security issues in Feel@Home are analysed, the security and privacy requirements are discussed, and the potential solutions are presented. As the example, the security building blocks are investigated to protect both IMS-based and VPN-based solutions.

The fifth paper, "Security Model for Emergency Real-Time Communications in Autonomous Networks" by (E. A. Panaousis et al. 2010), proposes a framework for emergency real-time communication using autonomous networks, called emergency Mobile Ad-hoc Networks (eMANETs). It targets for the needs of any society against any event that threatens serious damage to human welfare or to the environment. In this framework, a secure routing protocol, intrusion detection provision and security extension for real-time communications using peer-to-peer overlays are adopted. It is expect to be deployed in emergency cases where default telecommunications infrastructure has failed.

In the sixth paper, "Third Party Application Control on Quality of Service in IP Based Multimedia Networks" by (E. Pencheva and I. Atanasov 2010), the capabilities for open access to resource management that is one of the challenging issues in convergent and ubiquitous networks are investigated. By analysing the policy and charging control functions in Internet Protocol Multimedia Subsystem (IMS), the paper identifies the requirements for third party application control on quality of service (QoS). The Open Service Access (OSA) and Parlay $\mathrm{X}$ interfaces are designed and evaluated for supporting dynamic QoS control in IMS, and their implementation issues are discussed. This research topic is expected to attract more researchers in the near future.

The last paper, "Modeling the Influence of Network Delay on the User Experience in Distributed HomeAutomation Networks" by (M. Umberger et al. 2010), investigates the user-perceived quality of experience (QoE) in modern home-automation systems and services (HASS), which is also one of the key challenges in ubiquitous multimedia services. The subjective QoE assessment is proposed to measure the influence of network delay on HASS devices communication. Furthermore, the objective QoE assessment models are presented to represent the influence of network delay on a subjective QoE assessment for various HASS tasks. The users' satisfaction with respect to different network delays is tested by practical simulations. This paper mentions the novel research topic, and is expected to attract more readers.

Acknowledgments The guest editors wish to thank Prof. H. Raghav Rao and Prof. Ram Ramesh for providing the opportunity to edit this special issue on Ubiquitous Multimedia Services. We would also like to thank the authors for submitting their works as well as the referees who have critically evaluated the papers within the short stipulated time. Finally, we hope the reader will share our joy and find this special issue very useful.

\section{References}

Loukas, A., Damopoulos, D., Menesidou, S. A., Skarkala, M. E., Kambourakis, G., \& Gritzalis, S. (2010). MILC: A Secure and Privacy-Preserving Mobile Instant Locator with Chatting. Information Systems Frontiers.

Wang, T.-C., Wang, C.-H., Chang, R.-I., Liu, J.-C., \& Ho, J.-M. (2010). Effective distributed service architecture for ubiquitous video surveillance. Information Systems Frontiers.

Dong, Y., \& Lian, S. (2010). Automatic and fast temporal segmentation for personalized news consuming. Information Systems Frontiers.

Roman, R., Lopez, J., Dugeon, O., Lacaste, M., Tron, P. P., \& Bel, M. (2010). Advanced secure multimedia services for digital homes. Information Systems Frontiers.

Panaousis, E. A., Politis, C., Birkos, K., Papageorgiou, C., \& Dagiuklas, T. (2010). Security model for emergency real-time communications in autonomous networks. Information Systems Frontiers.

Pencheva, E., Atanasov, I. (2010). Third party application control on quality of service in IP based multimedia networks. Information Systems Frontiers.

Umberger, M., Lumbar, S., \& Humar, I. (2010). Modeling the influence of network delay on the user experience in distributed home-automation networks. Information Systems Frontiers. 
Shiguo Lian got his Ph.D. in multimedia security from Nanjing University of Science and Technology, China. He was a research assistant in City University of Hong Kong in 2004. He has been Research Scientist with France Telecom R\&D (Orange Labs) Beijing since July 2005. He authors or co-authors more than 90 refereed international journal/ conference papers and 16 patents. He has authored and edited 5 books, and contributed 16 chapters. His research interests include intelligent multimedia analysis and services, ubiquitous computing and communication, information and communication security. He got the Nomination Prize of "Innovation Prize in France Telecom", and he got "Top 100 Doctorate Dissertation in Jiangsu Province" in 2006. He has edited more than 10 special issues for international journals. He is a member of IEEE ComSoc Communications \& Information Security Technical Committee (CIS TC), IEEE Multimedia Communications Technical Committee (MMTC), and IEEE Technical Committee on Nonlinear Circuits and Systems (TC NCAS). He is the Area Editor of Journal of Signal Processing: Image Communication, Associate Editor of Security and Communication Networks, and Editorial Board member of Journal of Universal Computer Science (J.UCS). He has edited more than 10 special issues for international journals. He is the TPC members of IEEE ICC2008/2009/2010, IEEE GLOBECOM2008/2010, IEEE CCNC2009/ 2010, IWDW2008, etc., and invited reviewers of refereed journals and conferences.

Jean-Henry Morin holds a Ph.D. and a degree in Information Systems of the Economics and Social Sciences Faculty from University of Geneva where is Chargé de Cours in the Object Systems Group since 2000. He is now Associate Professor with University of Geneva, Switzerland. He is co-founder of PebbleAge SA a Geneva based company specialized in corporate performance management solutions where he was director of research and development until 2004. He has published in international conferences and journals and has worked on many European research projects. He is in the technical committee of some refereed conferences, such as The Third International Conference on Internet Monitoring and Protection (ICIMP 2008), and 2006 International Workshops on Databases, Information Systems, and Peer-to-Peer Computing (DBISP2P 2006). His research interests include Digital Rights and Policy Management (DRM/DPM), corporate information asset management, compliance, corporate governance, electronic commerce and services, Peer-to-Peer computing, mobile objects (agents), electronic publishing and information services over open networks. For some of his publications, see http://cui.unige.ch/ morin/.

Stefanos Gritzalis holds a BSc in Physics, an MSc in Electronic Automation, and a $\mathrm{PhD}$ in Information and Communications
Security from the Dept. of Informatics and Telecommunications, University of Athens, Greece. Currently he is the Deputy Head of the Department of Information and Communication Systems Engineering, University of the Aegean, Greece and the Director of the Laboratory of Information and Communication Systems Security (Info-Sec-Lab). He has been involved in several national and EU funded R\&D projects. His published scientific work includes 30 books or book chapters and more than 190 journal and international refereed conference and workshop papers. The focus of these publications is on Information and Communications Security and Privacy. His most highly cited papers have more than 700 citations (hindex=15). He has acted as Guest Editor in 16 journal special issues, and has leaded more than 30 international conferences and workshops as General Chair or Program Commitee Chair. He has served on more than 180 Program Committees of international conferences and workshops. He is an Editor-in-Chief or Editor or Editorial Board member for 13 journals and a Reviewer for more than 40 journals. He has supervised $9 \mathrm{PhD}$ dissertations. He was an elected Member of the Board (Secretary General, Treasurer) of the Greek Computer Society. His professional experience includes senior consulting and researcher positions in a number of private and public institutions. He is a Member of the ACM, the IEEE, and the IEEE Communications Society "Communications and Information Security Technical Committee". For more information, please see http://www.icsd.aegean.gr/sgritz.

Gregory L. Heileman received the BA degree from Wake Forest University in 1982, the MS degree in Biomedical Engineering and Mathematics from the University of North Carolina-Chapel Hill in 1986, and the $\mathrm{PhD}$ degree in Computer Engineering from the University of Central Florida in 1989. In 1990 he joined the Department of Electrical and Computer Engineering at the University of New Mexico, Albuquerque, NM, where he is currently Professor and Associate Chair. During 1998 he held a research fellowship at the Universidad Carlos III de Madrid, and in 2005 he held a similar position at the Universidad Politénica de Madrid. His research interests are in digital rights management, information security, the theory of computing and information, machine learning, and data structures and algorithmic analysis. He is the author of the text Data Structures, Algorithms and Object-Oriented Programming, published by McGraw-Hill in 1996. He is now leading the Digital Rights and Knowledge Engineering research group, focusing on the following areas: Intelligent Agents and Agentbased Negotiations, Digital Rights Management, Trust in Semantic Web and Social Networks, and Information Valuation and Personalization. For some of his publications, see http://www.eece.unm.edu/faculty/heileman/. 